\title{
Peptide-Mediated Targeting to Tumor Blood Vessels of Lung Cancer for Drug Delivery
}

\author{
Tong-Young Lee, ${ }^{1}$ Chin-Tarng Lin, ${ }^{1,2}$ Szu-Yao Kuo, ${ }^{3}$ De-Kuan Chang, ${ }^{1,3}$ and Han-Chung $\mathrm{Wu}^{1,3}$
}

${ }^{1}$ Institute of Pathology, College of Medicine, National Taiwan University; ${ }^{2}$ Department of Pathology, National Taiwan

University Hospital; and ${ }^{3}$ Institute of Cellular and Organismic Biology, Academia Sinica; Taipei, Taiwan

\begin{abstract}
Antiangiogenesis therapies for the treatment of cancers hold the promise of high efficacy and low toxicity. In vivo phage display was used to identify peptides specifically targeting tumor blood vessels. The peptide SP5-52 recognized tumor neovasculature but not normal blood vessels in severe combined immunodeficiency mice bearing human tumors. Synthetic peptide was shown to inhibit the binding of PC5-52 phage particles to the tumor mass in the competitive inhibition assay. Several selected phage clones displayed the consensus motif, proline-serine-proline, and this motif was crucial for peptide binding to the tumor neovasculature. SP552 peptides also bound vascular endothelial growth factorstimulated human umbilical vein endothelial cells and blood vessels of human lung cancer surgical specimens. Furthermore, this targeting phage was shown to home to tumor tissues from eight different types of human tumor xenografts following in vivo phage display experiments. An SP5-52 peptide-linked liposome carrying doxorubicin enhanced the therapeutic efficacy of the drug, markedly decreased tumor blood vessels, and resulted in higher survival rates of human lung and oral cancer-bearing xenograft mice. The current study indicates that ligand-targeted therapy offers improved therapeutic effects over conventional anticancer drug therapy, and that the peptide SP5-52 specifically targets tumor neovasculature and is a good candidate for targeted drug delivery to solid tumors. [Cancer Res 2007;67(22):10958-65]
\end{abstract}

\section{Introduction}

During tumor progression, tumor cells require increased supplies of oxygen and metabolites, and the efficient removal of waste products. In fact, establishing access to the host vascular system and the generation of a tumor blood supply are the ratelimiting steps of the tumor progression process (1). Tumor blood vessels are prime targets for therapies involving the inhibition of tumor growth, and these vessels express specific markers which are absent in the blood vessels of normal tissues. Many of these specific marker molecules, selectively expressed in tumor blood vessels, are proteins associated with tumor-induced angiogenesis, the sprouting of new blood vessels (2). These include the cell adhesion receptors, integrins, $\alpha_{\mathrm{v}} \beta_{3}$ and $\alpha_{\mathrm{v}} \beta_{5}$, which are overexpressed in the

Note: Supplementary data for this article are available at Cancer Research Online (http://cancerres.aacrjournals.org/).

T-Y. Lee, C-T. Lin, and S-Y Kuo contributed equally to this work

Requests for reprints: Han-Chung $\mathrm{Wu}$, Institute of Cellular and Organismic Biology, Academia Sinica, 128 Academia Road, Section 2, Nankang, Taipei 11529, Taiwan. Phone: 886-22789-9558; Fax: 886-22789-8059; E-mail: hcw0928@gate.sinica. edu.tw.

(C)2007 American Association for Cancer Research.

doi:10.1158/0008-5472.CAN-07-2233 tumor vasculature (3). Indeed, $\alpha_{v} \beta_{3}$ was recognized by an RGD peptide previously identified by in vivo phage display for tumor homing (4). Certain peptide ligands specific to these integrins have already been tested for the targeted delivery of anticancer and antiangiogenic agents (5). The finding of heterogeneity in the vasculature of normal and cancerous tissues provides promising opportunities for the targeted delivery of therapies.

Conventional chemotherapy is limited by the toxicity of the drugs to normal tissues. However, if these drugs could be preferentially directed to tumor sites or vessels instead of normal tissues, the toxic effects would be limited to the intended tumor tissue. Most small-molecule chemotherapeutic agents have a large volume of distribution on i.v. administration (6), often leading to a narrow therapeutic index due to the high toxicity to normal tissues. However, through encapsulation of the drugs in a macromolecular carrier, such as liposomes, the volume of distribution is significantly reduced and the concentration of drug in the tumor is increased (7). Liposomal doxorubicin represents a new class of chemotherapy delivery system that significantly improves the therapeutic index of doxorubicin in both preclinical (8-10) and clinical pharmacokinetics (11-13). This decreases the amount and types of nonspecific toxicity and increases the amount of drug that can be effectively delivered to the tumor site $(8,14)$.

A tumor-targeting ligand may be a more effective method of directing liposomes containing drugs to the tumor site, increasing the therapeutic efficacy of liposomal drugs. For solid malignancies, which comprise $>90 \%$ of human cancers, antibodies recognizing tumor-specific antigens have thus far provided little use for drug delivery because the immunoconjugates are too large to penetrate the tumor tissue $(15,16)$. However, phage-displayed peptide libraries have allowed the identification of small peptides a hundredth the mass of IgG antibodies, which might facilitate the development of such intervention strategies.

Phage-displayed random peptide libraries provide opportunities to map B-cell epitopes (17-20) and protein-protein contacts (21-23), and to select bioactive peptides bound to receptors $(24,25)$ or proteins $(22,26-28)$. They can be used to search for disease-specific antigen mimics (29-31), and to determine cellspecific $(32,33)$ and organ-specific peptides $(5,28,34)$. Screening phage-displayed peptide libraries against specific target tissues is therefore a direct and fast method for identifying peptide sequences which might be used for the targeting of drug or gene delivery vectors. In this study, we used a phage-displayed random peptide library to identify targeting peptides that could specifically bind to the tumor vessels of several tumor types. When coupled to liposomes containing the anticancer drug doxorubicin (Lipo-Dox), one targeting peptide, SP5-52, enhanced the efficacy of the drug against both human lung and oral cancer xenografts in severe combined immunodeficiency (SCID) mice. Our results indicate that this peptide has clinical potential as a drug delivery agent in the treatment of these tumors. 


\section{Materials and Methods}

Cell lines and cell culture. CL1-5 (lung cancer), H460 (lung cancer), and PC3 (prostate cancer) were grown in RPMI 1640 containing 10\% FCS (Life Technologies) at $37^{\circ} \mathrm{C}$ under a humidified atmosphere of $95 \%$ air and $5 \%$ $\mathrm{CO}_{2}$ (v/v). SAS (oral cancer), HCT116 (colon cancer), BT483 (breast cancer), Mahlavu (liver cancer), and $\mathrm{PaCa}$ (pancreatic cancer) were grown in DMEM (Life Technologies) containing 5\% FCS, in a $10 \% \mathrm{CO}_{2}$ incubator. CL1-5 was established by Chu et al. (35). H460, HCT116, BT483, PC3, Mahlavu, and $\mathrm{PaCa}$ were obtained from the American Type Culture Collection. Human umbilical vein endothelial cells (HUVEC) were isolated from the umbilical vein after separation from the placenta, and grown in M199 medium (Life Technologies) supplemented with $20 \% \mathrm{FCS}$ and $15 \mu \mathrm{g} / \mathrm{mL}$ of endothelial cell growth supplement (Upstate) in a $5 \% \mathrm{CO}_{2}$ incubator.

In vivo phage display biopanning procedures. SCID mice 4 to 6 weeks of age were injected s.c. into the dorsolateral flank with CL1-5 cells to produce lung cancer xenografts. A phage-displayed peptide library (New England Biolabs, Inc.), which initially contained $5 \times 10^{10}$ plaque-forming units (pfu), was injected through the tail vein of SCID mice bearing sizematched CL1-5-derived tumors $\left(\sim 500 \mathrm{~mm}^{3}\right)$. After $8 \mathrm{~min}$ of phage circulation, the mice were treated with diethyl ether to drive them into deep anesthesia, and perfused with $50 \mathrm{~mL}$ of PBS to wash out unbound phage. The control organs (lungs, heart, brain) and tumor masses were removed, weighed, and washed with cold PBS. The organs and tumor samples were homogenized and the phage particles were rescued by ER2738 bacteria (New England Biolabs). The phages were titered on agar plates in the presence of $1 \mathrm{mg} / \mathrm{L}$ of IPTG/X-gel. Bound phages were amplified and subjected to four consecutive rounds of biopanning using SCID mice bearing lung cancer xenografts prepared as described above, except that a phage input of $2 \times 10^{11}$ pfu was used. The phages eluted from the fifth round were titered on LB/IPTG/X-Gal plates. The candidate tumor-homing phage clones were randomly selected and identified by cellular ELISA and in vivo homing experiments.

DNA sequencing and computer analysis. DNA sequences of the selected phages were determined according to the dideoxynucleotide chain termination method using an automated DNA sequencer (ABI PRISM 377; Perkin-Elmer). Sequencing was done with the primer 5 -CCCTCATAGTTAGCGTAACG-3' corresponding to the pIII gene sequence. The phagedisplayed peptide sequences were translated and aligned using the Genetics Computer Group program.

Peptide synthesis. The peptides, SVSVGMKPSPRP (SP5-52), SVSVGMKGGGRP (MP5-52), and control peptide RLLDTNRPLLPY were synthesized and purified by reverse-phase high-performance liquid chromatography to $>95 \%$ purity by Invitrogene, Inc. Conjugation of these peptides with FITC or biotin was done by the same company by adding FITC or biotin to the $\mathrm{NH}_{2}$ terminus.

In vivo homing experiments and peptide binding in xenograft tumor vessels. Phage clones $\left(10^{9} \mathrm{pfu}\right)$ or control helper phage (insertless phages) were injected into the tail vein of tumor xenograft mice which were then kept for $8 \mathrm{~min}$. After perfusion, xenograft tumors and organs were removed and titered. In peptide-competitive inhibition experiments, phage clones were coinjected with $100 \mu \mathrm{g}$ of synthetic peptide. After injection of targeting or control phage clones, the organs and tumors were removed and divided into two parts. One part was titered by ER2738, and another part was embedded in optimal cutting temperature (Tissue-Tek). The optimal cutting temperature-embedded frozen tissues were sectioned at $5 \mu \mathrm{m}$ and transferred to cold PBS buffer. The sections were fixed with acetone/ methanol (1:1), washed with PBS and immersed in blocking buffer (1\% bovine serum albumin in PBS) for $1 \mathrm{~h}$. Then, the sections were incubated with rat anti-mouse CD31 (BD PharMingen), rabbit anti-rat antibody (Stressgen, Canada) and immersed in rhodamine-labeled goat anti-rabbit antibody (Jackson ImmunoResearch). The slides were further incubated with mouse anti-M13 antibody (Amersham Biosciences), followed by FITClabeled goat anti-mouse antibody (Jackson ImmunoResearch), and immersed in Hoechst 33258 (Molecular Probes). Finally, slides were washed and mounted with mounting medium (Vector). In the same way, we injected FITC-labeled targeting peptides and control peptide in place of the phages, into tumor xenograft mice for a peptide-binding assay. After perfusion, tissues were removed and treated as above. Slides were examined under a Leica Universal microscope. Images were merged by SimplePCI software (C-IMAGING).

Targeting peptide binding to vascular endothelial growth factorstimulated HUVECs and lung cancer biopsy specimens. HUVECs were plated and grown to $\sim 80 \%$ confluence on coverslips. The cells were pretreated with $20 \mathrm{ng} / \mathrm{mL}$ of vascular endothelial growth factor (VEGF; R\&D Systems) and $2 \mathrm{ng} / \mathrm{mL}$ of basic fibroblast growth factor (Peprotech) for $48 \mathrm{~h}$ The VEGF-stimulated HUVECs were washed with serum-free M199 plus 3\% BSA, incubated in blocking buffer for $30 \mathrm{~min}$ at $4{ }^{\circ} \mathrm{C}$ and then with phages at $4^{\circ} \mathrm{C}$ for $1 \mathrm{~h}$. They were washed and fixed with $3 \%$ paraformaldehyde for $10 \mathrm{~min}$, followed by incubation with mouse anti-M13 monoclonal antibody (Amersham Biosciences) for $1 \mathrm{~h}$, and then FITC-labeled anti-mouse antibody (Jackson ImmunoResearch), followed by staining in Hoechst 33258. The coverslips were finally washed and mounted.

For localization of the peptide-binding ability on lung cancer tissue, frozen sections of human lung adenocarcinoma were incubated with biotinlabeled peptide using routine immunohistochemical procedures. The frozen surgical specimens were obtained from the tissue bank of National Taiwan University Hospital with approval from the Institutional Review Boards (IRB9461702021).

Preparation of peptide-liposome containing doxorubicin. Procedures for the preparation of peptide-liposomes containing doxorubicin were adapted from the methods published in previous reports $(33,36)$. Briefly, peptide ligands were coupled to NHS/PEG/DSPE [ $N$-hydroxysuccinimidocarboxyl/polyethylene glycol (PEG; average molecular weight, 3,000)derived/distearoylphosphatidylethanolamine; NOF Corporation] in a 1:1.5 molar ratio. The reaction was completed and confirmed by quantitation of the remaining amino groups, measured using a trinitrobenzenesulfonate reagent (37). Peptidyl-PEG/DSPE was transferred to preformed liposomes after coincubation at a temperature above the transition temperature of the lipid bilayer (38). The liposomes had a particle size ranging from 65 to $75 \mathrm{~nm}$ in diameter (33). There were 300 to 500 peptide molecules per liposome, computed as described previously (39).

Animal model for study of ligand-targeted therapy. Human lung (CL1-5) and oral (SAS) cancer xenografts were established in SCID mice. Mice 4 to 6 weeks of age were injected s.c. into the dorsolateral flank with human cancer cells. Mice with size-matched tumors (tumor sizes of $\sim 100 \mathrm{~mm}^{3}$ ) were randomly assigned to different treatment groups and treated with SP5-52-Lipo-Dox, Lipo-Dox, or equivalent volumes of PBS i.v. at a total doxorubicin dose of $7 \mathrm{mg} / \mathrm{kg}$ (1 mg/kg, twice a week). Mouse body weight and tumor size were measured twice a week using calipers. The tumor volumes were calculated using the equation: length $\times(\text { width })^{2} \times$ 0.52. The differences in mean tumor volume were evaluated by ANOVA Animal care was carried out in accordance with the guidelines of Academia Sinica, Taipei, Taiwan

\section{Results}

Recovery of phage-displayed tumor-homing peptides from human carcinoma xenografts. To isolate tumor-homing phages from tumor tissues, we used a phage-displayed peptide library to treat the NSCLC (non-small cell lung cancer; CL1-5) tumor-bearing mice for five rounds of in vivo selection (biopanning). The recovery rate of the fifth round of biopanning had increased 156-fold over that of the first round (Fig. $1 A$ ). Sixty enriched phages from the fifth round were randomly selected and sequenced. Fifty-five clones had their displayed peptide sequences confirmed. Using a Genetics Computer Group software, the three most frequent clones (PC5-5, -52, and -54) were shown to have the consensus motif of proline-serine-proline (PSP). Four clones (PC5-7, -8, -10, and -50) had consensus amino acid residues, proline-serine (PS), whereas another four (PC5-10, -53, -58, and -60) had the consensus amino acid residues, serine-proline (SP; Table 1). The frequency of PC5-52 increased to $45 \%$ (25 of 55) in the fifth cycle of biopanning 


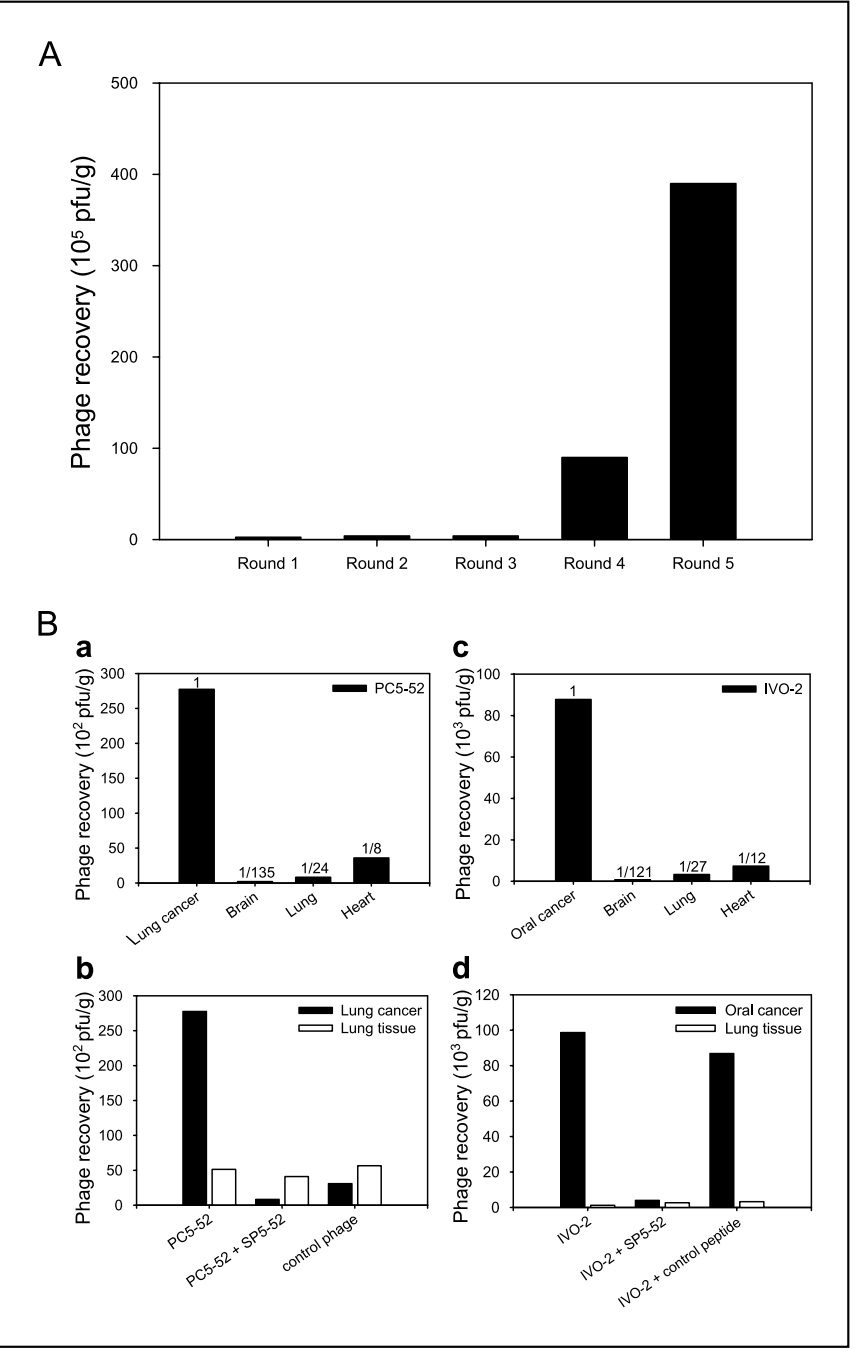

Figure 1. Selection of lung cancer-targeting phages by in vivo phage display $A$, a phage-displayed peptide library was injected through the tail vein of tumor-bearing mice. Phage recovered from the tumor was amplified and re-injected in four consecutive rounds. After the fifth round of biopanning (lane 5), the recovery rate of the phages had increased 156-fold over that of the first round. $B$, identification of specificity of tumor-homing phage. a, SCID mice bearing human lung cancer xenografts were injected i.v. with PC5-52 phage, and phage was recovered after perfusion. Recovery of PC5-52 phage from the tumor was higher than from brain, lungs, and heart. Phage titer in control organs compared with tumor tissues is indicated. $b$, recovery of lung cancer-homing phage was competitively inhibited by the synthetic peptide SP5-52. A background level of phage titer was recovered from the tumor mass of mice treated with a control phage. $c$, SCID mice bearing human oral cancer xenografts were injected i.v. with IVO-2 phage, and phage was recovered after perfusion. The results show that IVO-2 phage exhibits marked homing to the tumor compared with the control organs (brain, lungs, and heart) in the same mouse. Phage titer in control organs compared with tumor tissues is indicated. $d$, recovery of oral cancer-homing phage is competitively inhibited by the synthetic peptide SP5-52. However, control peptides have no such effect.

(Table 1). Interestingly, we also identified this sequence, SVSVGMKPSPRP (SP5-52), in a separate study using oral cancer for in vivo phage display, on a phage we named, IVO-2 (Table 1; Fig. 1B-c). IVO-2 was isolated from oral cancer xenografts by in vivo phage display and displayed the same amino acid sequence as PC5-52. Due to its high frequency and the possibility of universal expression in tumor vessels, we focused on this peptide for further study.

Identification of tumor-homing phage. To investigate the targeting ability of PC5-52, phages were injected into the tail vein of mice bearing lung cancer cell-derived tumors and then recovered after perfusion. The titers of the phages in the tumor mass and in other normal organs were determined. PC5-52 showed tumorhoming ability, exhibiting a concentration from 8.0- to 135-fold higher in the tumor mass than in normal organs, including the brain, lungs, and heart (Fig. $1 B-a$ ), whereas the control helper phage revealed no such homing ability (Fig. $1 B-b$ ). This was also proved by a ligand competition experiment which showed that coinjection of synthetic SP5-52 peptide with PC5-52 phage particles inhibited the recovery of PC5-52 from tumor tissues (Fig. $1 B-b$ ). Coinjection of $100 \mu \mathrm{g}$ of SP5-52 inhibited $97 \%$ of PC5-52 homing to the NSCLC tumor mass. Similarly, IVO-2 phage particles (which display a peptide with the same amino acid sequence as that displayed by PC5-52) also homed to the tumor tissues of oral cancer xenografts (Fig. 1B-c). IVO-2 showed a 12- to 121-fold higher concentration in tumors than in organs such as normal brain, lungs, and heart (Fig. $1 B-c$ ). The tumor-homing ability of IVO-2 could also be inhibited by SP5-52. Coinjection of $100 \mu \mathrm{g}$ of SP5-52 inhibited $95.9 \%$ of IVO-2 homing to oral cancer xenografts (Fig. $1 B-d$ ). Control peptides did not inhibit IVO-2 homing to tumor tissue (Fig. 1 $B-d$ ). Twenty-four hours after the injection of PC5-52, these phages had accumulated 10 -fold more in tumor tissue than in control tissues (data not shown).

The tumor-homing peptide targets tumor neovasculature. To investigate the homing specificity of PC5-52, we used immunofluorescent staining to identify the binding site of the phage after perfusion. Phage particles reacted with xenograft lung cancer tumor tissues but not with normal lung tissues (Fig. 2A). However, phages did not localize to cancer cells. Using frozen sections from tumor tissues incubated with anti-M13 monoclonal antibody (green) and mouse endothelial cell marker CD31 (red), PC5-52 was found to be colocalized with CD31 in the tumor neovasculature of xenograft tumor tissues (Fig. $2 B, i-k$ ). The phage was not found in vessels of normal heart (Fig. $2 B-e$ ), lungs, and brain (data not shown).

Table 1. Alignment of phage-displayed peptide sequences selected from lung cancer cell xenografts

Phage clone

Phage-displayed peptide sequence*

Frequency

\begin{tabular}{lcr}
\hline PC5-52 & SVSVGMKPSPRP & $25 / 55^{\dagger}$ \\
PC5-54 & WPLHTSVYPPSP & $11 / 55$ \\
PC5-5 & NTLPPFSPPSPP & $5 / 55$ \\
PC5-10 & SFPDSNIAPSSP & $3 / 55$ \\
PC5-8 & QHAPSNSKSVLT & $2 / 55$ \\
PC5-7 & WPTYLNPSSLKA & $1 / 55$ \\
PC5-57 & GPSGNLHIRPAS & $1 / 55$ \\
PC5-53 & SPLLSTRAVQLS & $1 / 55$ \\
PC5-58 & SPMFTMIQGDAQ & $1 / 55$ \\
PC5-60 & VNSHQALWSPAQ & $1 / 55$ \\
PC5-4 & STLPPPLRFANV & $1 / 55$ \\
PC5-2 & SFNQPYLYKTAF & $1 / 55$ \\
PC5-3 & YHTRIALPDNLP & $1 / 55$ \\
PC5-11 & AQSTAFQKPLLM & $1 / 55$ \\
& &
\end{tabular}

*Phage-displayed consensus amino acids are shown in boldface. †From 60 random selected phage clones, 55 phage-displayed peptide sequences were identified and aligned. 
Figure 2. Localization of phages displaying tumor-homing peptide. $A$, immunofluorescent localization of PC5-52 after i.v. injection into human lung cancer bearing mice. The immunoreactivity of phages are localized in tumor masses (a), whereas no reaction product is observed in normal lungs $(c) . b$ and $d$, cells counterstained for nuclear DNA with $\mathrm{H} 33258$ corresponding to $a$ and $c$, respectively (bar, $20 \mu \mathrm{m}$ ). $B$, investigation of tumor-homing phage binding to tumor vessels of lung cancer xenograft. Mice were injected with tumor-homing phages by i.v. injection. Tumor and organ tissues were immunostained with anti-M13 and anti-CD31 antibodies. Anti-phage immunofluorescence is colocalized with anti-CD31 on tumor neovasculature endothelia after phage injection $(i, j$, and $k)$. Control phage cannot bind to tumor vessels (a). The tumor-homing phage cannot recognize the normal heart (e). Anti-CD31 in heart vessels ( $f$ and $g$ ) and xenograft tumor vessels $(b, c, j$, and $k)$. Nuclear staining by $\mathrm{H} 33258(d, h$, and $l)$. $C$, binding of FITC-labeled SP5-52 peptide to tumor vessels of lung cancer xenografts.

FITC-labeled SP5-52 could bind to human lung cancer xenograft sections $(i)$ and colocalizes with anti-CD31 on tumor neovasculature $(k)$. An FITC-labeled control peptide did not bind lung cancer xenografts (a) and SP5-52 did not bind to a control heart tissue $(e)$. Anti-CD31 in the human lung cancer xenografts ( $b$ and $j$ ) and normal heart $(f)$ vessels. Nuclear staining by $\mathrm{H} 33258$ in $d, h$ and I (bar, $20 \mu \mathrm{m}$ ).

\section{A}

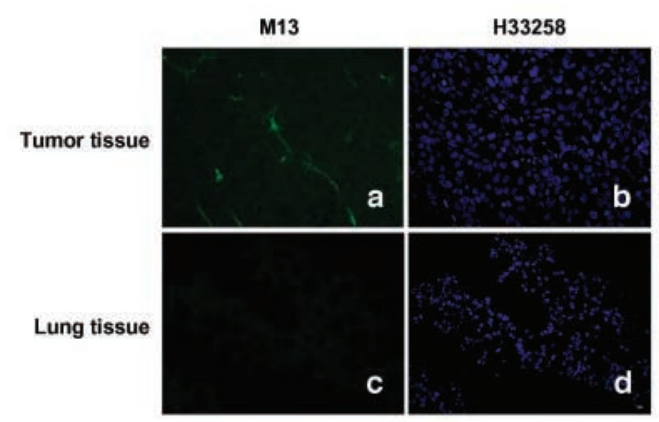

B
B
Control phage
tumor tissue

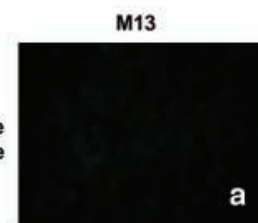

a

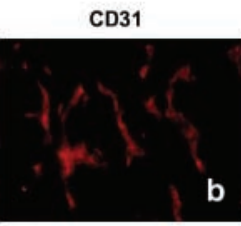

b
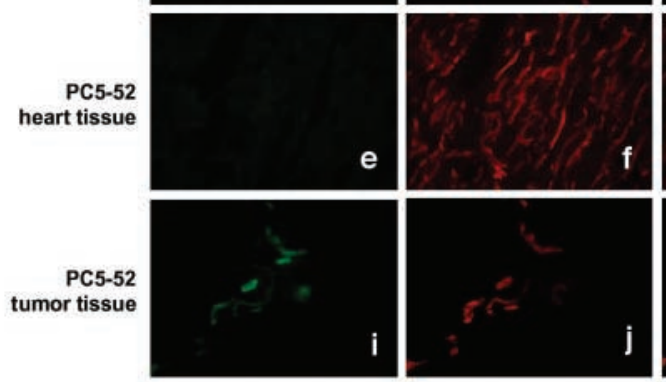

C
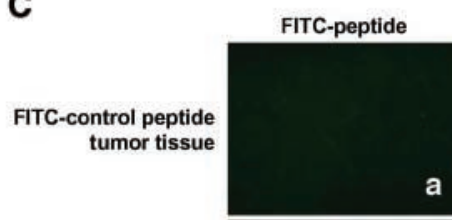

a
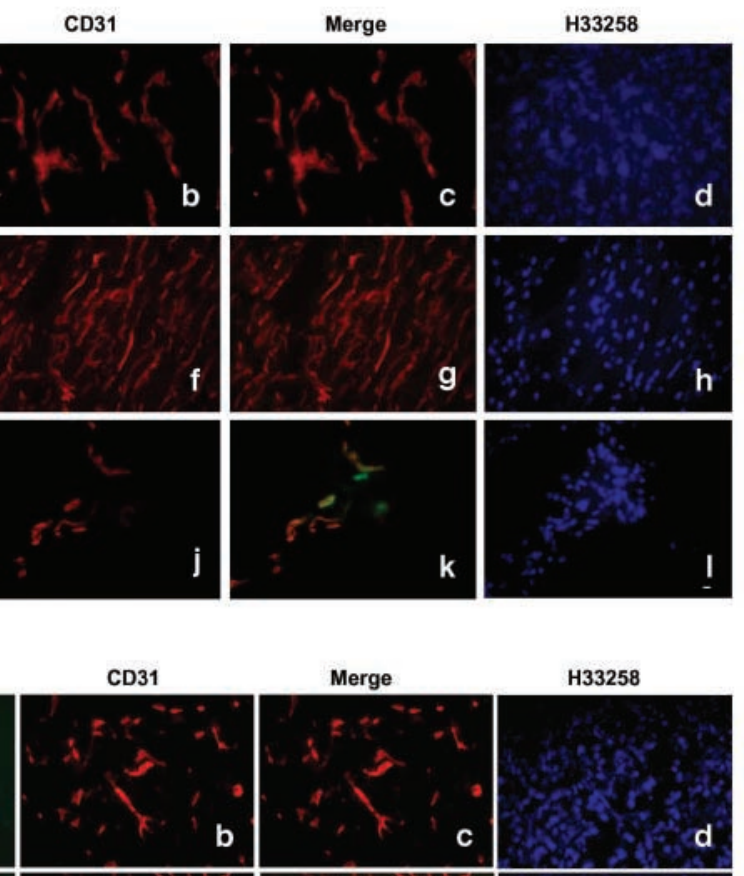

H33258
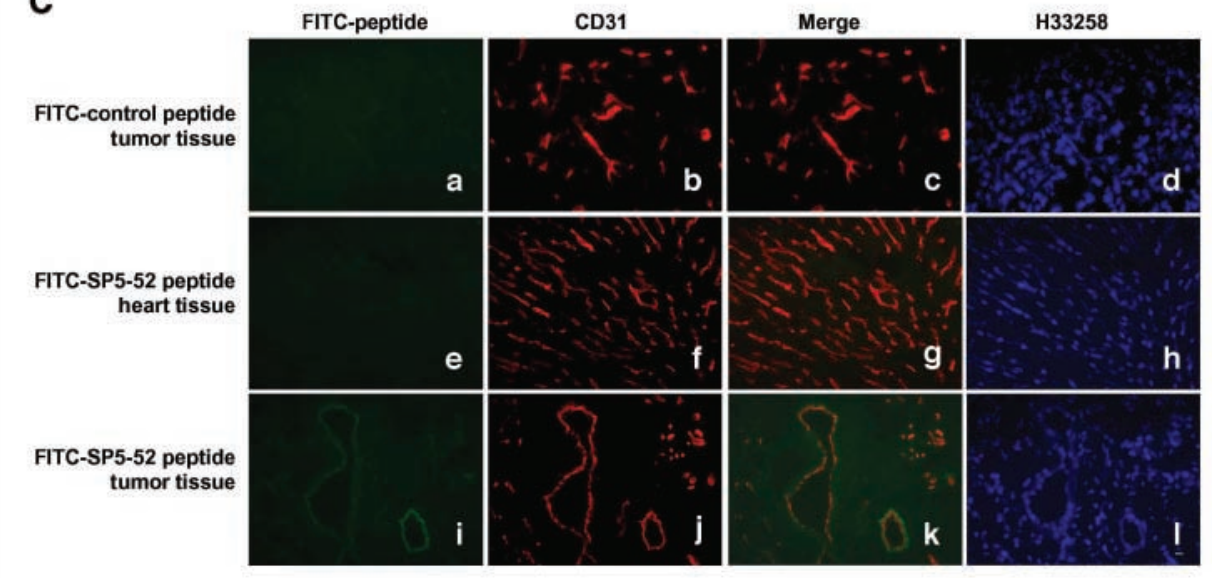

To further investigate phage-displayed peptide homing to the tumor neovasculature, we used an FITC-labeled SP5-52 peptide in place of the PC5-52 phage. FITC-labeled SP5-52 peptides also colocalized with mouse CD31 marker in the tumor neovasculature of lung cancer xenografts (Fig. $2 C, i-k$ ). They were not detected in normal heart vessels (Fig. $2 C, e-g$ ). FITC-labeled control peptides did not recognize tumor neovasculature (Fig. 2C, $a-c$ ).

Phages displaying the SP5-52 sequence were isolated from lung and oral cancer xenografts by in vivo phage display. To test the hypothesis that the target of SP5-52 may be universally expressed in the neovasculature of solid tumors, we examined the homing ability of PC5-52 in six other types of human cancers including human lung (H460), colon (HCT116), breast (BT483), prostate (PC3), liver (Mahlavu), and pancreatic (PaCa) cancer xenografts. Interestingly, in all of these human cancer xenografts, PC5-52 targeted tumor tissues but not normal organs like brain, heart, and lungs (Fig. $3 A$ ). Control phage without this targeting ligand had no such homing ability (data not shown).

From in vivo phage display, we identified three clones (PC5-5, -52, and -54) with a PSP consensus motif (Table 1). We proposed that these three amino acid residues play a crucial role in homing to tumor tissues. To test this hypothesis, we changed these three amino acid residues in SP5-52 (SVSVGMKPSPRP) to GGG in a mutant peptide, MP5-52 (SVSVGMKGGGRP). PC5-52 targeted 
CL1-5-derived tumors in an in vivo homing assay (Fig. 3B-a) and this tumor-homing ability was markedly inhibited by the peptide, SP5-52 (Fig. 3B-b). However, competitive inhibition was lost when the consensus motif PSP of SP5-52 had been changed to GGG in MP5-52 (Fig. 3B-c).

PC5-52 reacts with stimulated HUVECs and human lung cancer biopsy specimens. We showed that PC5-52 targets the mouse neovasculature endothelia of tumor tissues. To identify whether this phage has affinity for human neovasculature endothelia, we applied the PC5-52 phage particles to VEGFstimulated HUVECs. PC5-52 bound to VEGF-stimulated HUVECs (Fig. 4A-a), whereas the control phage without this peptide showed no binding activity (Fig. 4A-b). HUVECs without VEGF stimulation did not display PC5-52 binding (Fig. 4A-c). SP5-52 (100 $\mu \mathrm{g} / \mathrm{mL})$ could compete with PC5-52 for binding to stimulated HUVECs (Fig. $4 A-d$ ), but the same concentration of mutated peptide, MP5-52, could not (Fig. 4A-e). Biotin-labeled SP5-52 peptide bound to tumor vessels of human lung cancer surgical specimens but not to normal pulmonary vasculature (Fig. $4 B, a$ and $c$ ). Anti-CD31 antibody bound both tumor and normal blood vessels (Fig. $4 B, b$ and $d$ ). Sixty percent ( 6 of 10 ) of pulmonary adenocarcinomas from 10 patients expressed a target protein recognized by the peptide (Fig. 4B; Supplementary Fig. S1). PC5-52 binding to human NSCLC surgical specimens was inhibited by $100 \mu \mathrm{g} / \mathrm{mL}$ of SP5-52 (Supplementary Fig. S1e and $f$ ). These data indicate that SP5-52 peptide could recognize unidentified receptors on human angiogenic endothelial cells, and on the tumor neovasculature of both humans and mice.

Animal model for study of ligand-targeted therapy. To determine whether SP5-52 could be used to improve the efficacy of cancer chemotherapeutics, we coupled SP5-52 to liposomes containing the antiangiogenic drug, doxorubicin (SP5-52-Lipo-Dox). SCID mice bearing a CL1-5 xenograft (tumor size $\sim 100 \mathrm{~mm}^{3}$ ) were then treated with SP5-52-Lipo-Dox, Lipo-Dox, or PBS. The SP5-52Lipo-Dox-treated mice showed a significantly smaller tumor size than Lipo-Dox- and PBS-treated cases $(P<0.01$; Fig. $5 A)$. The tumor size of the Lipo-Dox group gradually increased to 1.9-fold larger than that of the SP5-52-Lipo-Dox group by day 28 . The tumor size of the control PBS group was 4.1-fold larger than that of the SP5-52-Lipo-Dox group (Fig. $5 A$ ). The body weights of each group showed no significant changes (data not shown). To further characterize therapeutic efficacy, we compared the animal survival rates after 21 days of treatment. When we stopped the experiment on day 81 , all animals $(n=6)$ had died in the PBS and Lipo-Dox groups ( $0 \%$ survival rate), whereas only two animals had died in the SP5-52-Lipo-Dox group (66.7\% survival rate; Fig. $5 B$ ). To verify the antiangiogenic effect of SP5-52-Lipo-Dox, we repeated the above experiment and removed the tumor tissues for analysis of the effect on tumor blood vessels. Tumor vessels were found to be markedly decreased in SP5-52-Lipo-Doxtreated mice but not in mice of the PBS groups. Lipo-Dox-treated mice showed a limited reduction in the tumor vasculature (Supplementary Fig. S2).

To test whether SP5-52 could also increase the therapeutic efficacy for oral cancer, we administered ligand-targeted therapy for this cancer in an animal model. SCID mice bearing oral cancer xenografts were treated with SP5-52-Lipo-Dox, Lipo-Dox, or PBS. Similar results were found. The SP5-52-Lipo-Dox-treated mice showed a significantly smaller tumor size than Lipo-Dox and PBStreated mice $(P<0.05$; Fig. $5 C)$. The tumor size in the Lipo-Dox

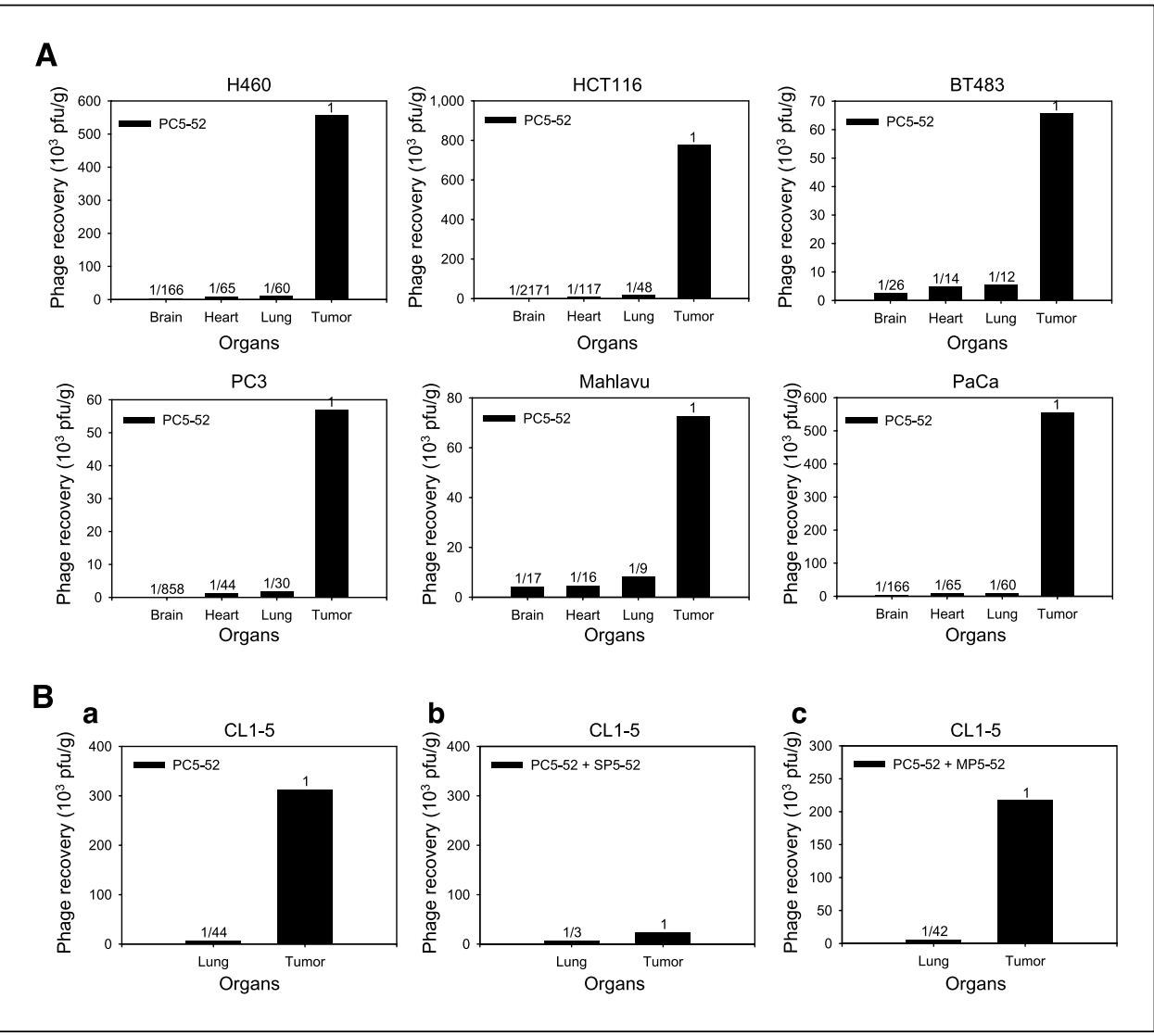

Figure 3. PC5-52 phage homes to a variety of human cancer xenografts, directed by a three-amino acid motif. $A$, recovery of tumor-targeting phage PC5-52 from SCID mice bearing different human cancer xenografts. SCID mice bearing human lung $(\mathrm{H} 460)$, colon (HCT116), breast (BT483), prostate $(P C 3)$, liver (Mahlavu) and pancreatic $(\mathrm{PaCa})$ cancer xenografts were injected i.v. with PC5-52. After washing out free phages, PC5-52 phage titer in the tumor masses were found to be markedly higher than in control organs, brain, lungs, and heart in all the human cancer xenografts. $B$, identification of binding motif. SCID mice bearing human lung cancer xenografts were injected i.v. with PC5-52 phage $(a)$ or phage plus SP5-52 (b) or mutated peptide MP5-52 (c). The phages from control lung and tumor tissues were recovered after perfusion. Recovery of lung cancer homing phage is competitively inhibited by the synthetic peptide SP5-52 (b). MP5-52 has lost this competition ability $(c)$. Phage titers in control organs compared with tumor tissues. 
Figure 4. Targeting phages react with VEGF-stimulated HUVECs and bind to endothelial cells of human tumors. $A$, PC5-52 phages were incubated with VEGF-stimulated HUVECs. Unbound phage particles were washed off and the HUVECs immunostained with FITC anti-M13 antibodies. The specific reactivity of PC5-52 on stimulated HUVECs (a). The control helper phage does not react with stimulated HUVECs (b). PC5-52 exhibits no specific reactivity with nonstimulated HUVECS $(c)$. SP5-52 (d) but not MP5-52 (e) can compete with PC5-52 for binding to stimulated HUVECs. Nuclear staining was by $\mathrm{H} 33258$ (bar, $20 \mu \mathrm{m}$ ). $B$, immunohistochemical localization of biotin-labeled SP5-52 on human lung adenocarcinoma specimens. The biotin-labeled peptide was incubated with frozen sections of surgical specimens and subjected to routine immunohistochemical staining. The targeting peptide was detected in tumor tissue (a), but not in non-tumor lung tissue (c). CD31 was detected both in vessels of tumor tissue $(b)$ and in control lung tissue $(d)(b a r, 20 \mu \mathrm{m})$.
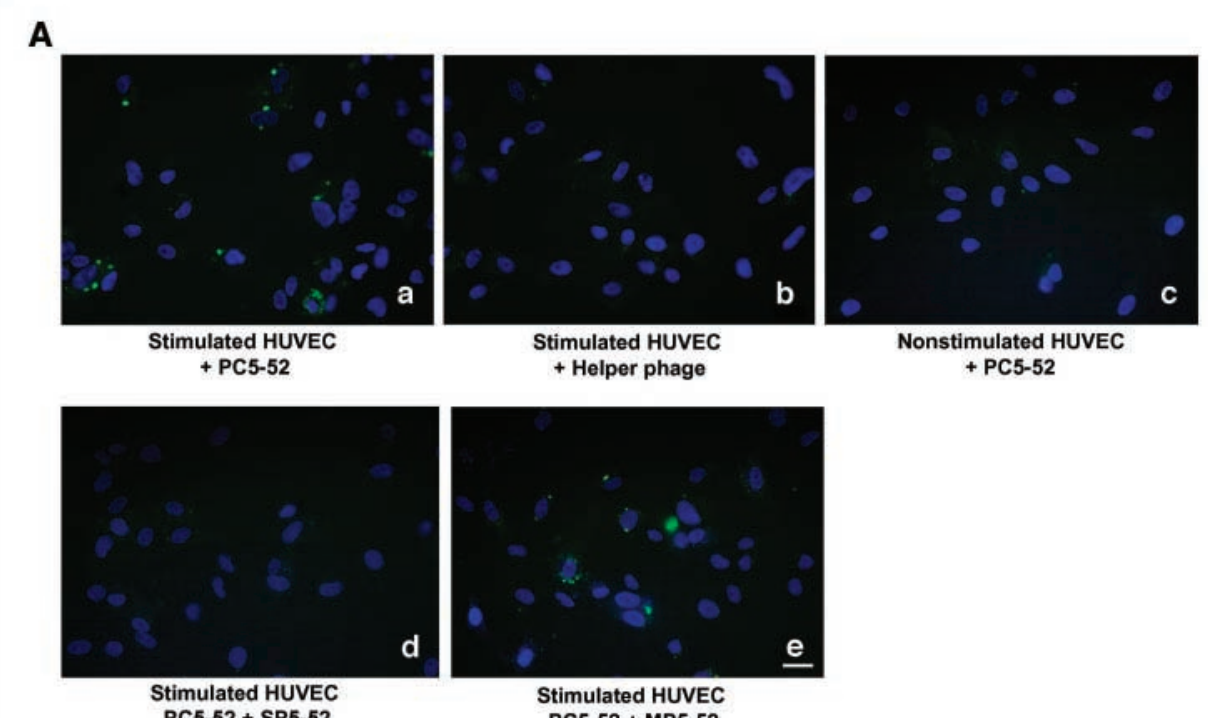

Stimulated HUVEC

PC5-52 + MP5-52

B

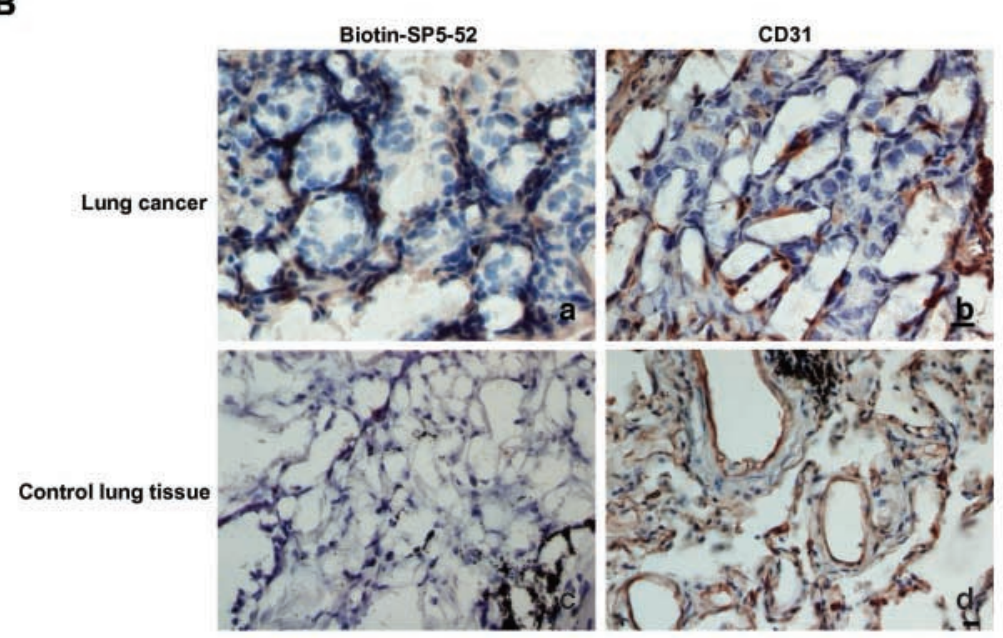

group gradually increased to 3.6-fold larger than that of the SP5-52Lipo-Dox group on day 24.5. Mice in the control PBS group were found to have a tumor size 7.1-fold larger than that of the SP5-52Lipo-Dox group (Fig. $5 C$ ). The body weights of each group showed no significant changes (data not shown). To further characterize therapeutic efficacy, we compared animal survival rates after 21 days of treatment. All animals $(n=6)$ had died in the PBS group ( $0 \%$ survival rate), four animals had died in the Lipo-Dox group (33.3\% survival rate), whereas all the SP5-52-Lipo-Dox-treated mice had survived ( $100 \%$ survival rate) when the experiment had finished on day 60 (Fig. 5D). Repeating this experiment further confirmed that SP5-52-Lipo-Dox was more effective than Lipo-Dox in treating oral cancer xenografts, with the tumor size of the LipoDox group gradually increasing to 3.2-fold that of the SP5-52-LipoDox group on day 24.5. The control PBS group had a tumor size 5.9-fold larger than that of the SP5-52-Lipo-Dox group (data not shown). Thus, conjugation of Lipo-Dox with the targeting ligand SP5-52 enhances the ability of the drug to inhibit the growth of solid tumors, including human lung and oral cancer xenografts in SCID mice.

\section{Discussion}

Most cancer chemotherapy is accompanied by strong side effects and acquired drug resistance. Therefore, there is a pressing need for drug delivery systems to deliver drugs to target sites on both tumor vessels and tumor cells. Some attempts have been made, including the use of antibodies (40) and targeting peptides (33) against tumor cells. Vascular targeting is also a focus of interest, because angiogenesis is a required step for tumor expansion, and drugs or drug carriers may first meet neovasculature before extravasation in the tumor (41). Because tumor vasculature expresses unique markers that distinguish it from normal vasculature (42), targeting to markers of tumor angiogenic vasculature is a promising strategy for cancer treatment. In this study, we used in vivo phage display to identify tumor homing peptides, including SP5-52, which could specifically bind not only the tumor vessels of xenografts in animal models, but also VEGFstimulated HUVECs and human lung cancer vessels. Such peptides are useful tools for active targeting to tumor neovasculature.

In vivo biopanning identified phages (such as PC5-52) with high binding activity to lung cancer xenografts. Phages from the fifth 
round showed 156-fold higher affinity than those from the initial round (Fig. $1 A$ ) with several of the phage clones displaying the PSP consensus peptide motif (Table 1). The binding activity of PC5-52 with tumor tissues could be inhibited by the synthetic peptide, SP5-52 (Fig. $1 B, b$ and $d$ ) indicating that the synthetic peptides bind the same sites as their respective phage clones. That the mutated synthetic peptide, MP5-52, could not inhibit PC5-52 binding shows the importance of the PSP motif (Fig. 3B). Moreover, this phenomenon was observed both in mouse xenograft tumors and in human-stimulated HUVEC cells (Fig. 4A). Closer examination revealed that PC5-52 phage and SP5-52 peptide colocalized with CD31 in most xenograft tumor vascular endothelia but were rarely detected in tumor cells or normal organs (Fig. 2). Our findings indicate that PC5-52 could specifically target tumor neovasculature endothelial cells, but not the vasculature of normal organs, and that the PSP motif is required for this targeting.

IVO-2 and PC5-52 carried the same peptide, and we determined that the peptide sequence could direct the phages to the vasculature of xenografts derived from other human tumor cells. Xenograft tumor tissues from eight different cancer cell lines contained $>8$-fold more PC5-52 than normal organs (Figs. $1 B$ and $3 A$ ), indicating a higher affinity of PC5-52 for these tumor tissues. These phenomena suggest that the neovasculature in solid tumors may universally express an unidentified receptor not expressed in normal mature vasculature, and that this receptor recognizes the SP5-52 peptide.

This peptide, selected using the murine angiogenic model, had the same binding affinity for the neovasculature endothelia in human tumors, and was able to specifically bind both human lung cancer biopsy specimens and VEGF-stimulated endothelial cells (HUVECs; Fig. 4). Therefore, the molecular target may be specifically expressed in angiogenic vessels. This putative receptor could recognize a peptide ligand with the sequence,
SVSVGMKPSPRP. A Blast search for this 12-mer peptide revealed PSP motif homologies to seven human sequence entries. However, although SP5-52 possesses homology with predicted protein interaction and DNA binding motifs in human proteins, the PSP motif per se was not identified as a known ligand for membrane proteins.

Our finding that this peptide targets the neovasculature indicates a high potential utility in tumor detection and directed chemotherapeutic drug delivery. Using SP5-52-conjugated LipoDox to compare the effects with nontargeted Lipo-Dox on tumor growth, we showed that SP5-52-Lipo-Dox enhanced the efficacy of the drug against human lung and oral cancer xenografts (Fig. $5 \mathrm{~A}$ and $C$ ). The targeting liposome also significantly increased the survival rate in these two human cancer xenograft animal models (Fig. $5 B$ and $D$ ). This targeting strategy has significant clinical potential to improve cancer treatment.

The tumor vasculature is a particularly suitable target for cancer therapy because it is composed of genetically stable nonmalignant endothelial cells that induce little or no drug resistance (43). In addition, these cells are more accessible to drugs and have an intrinsic amplification mechanism. It has been estimated that elimination of a single endothelial cell could inhibit the growth of 100 tumor cells $(44,45)$. The severe damage to tumor vasculature caused by SP5-52-Lipo-Dox throughout the tumors (Supplementary Fig. S2) clearly shows an improvement in chemotherapeutic efficacy in a xenograft animal model. This may be due to an increased accumulation of targeted liposomes in tumor tissues. The high tumor interstitial fluid pressure (IFP) of solid tumors is a barrier to efficient drug delivery (46); increased IFP contributes to decreased transcapillary transport in tumors, which leads to the decreased uptake of drugs and eventual failure of therapy. These phenomena are accompanied by the development of drug resistance and metastatic disease (46-50). The ligand-targeted

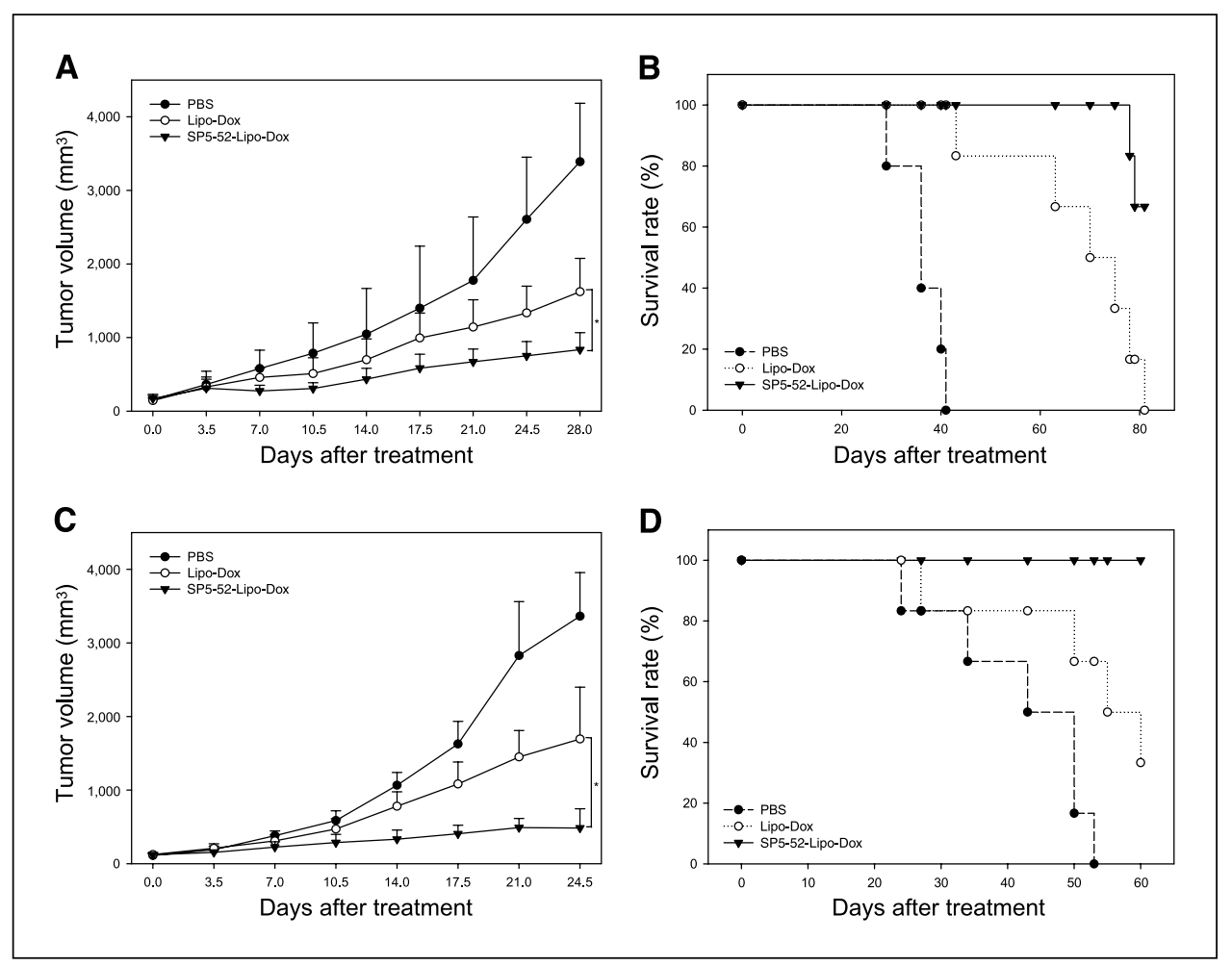

Figure 5. Treatment of SCID mice bearing human cancer xenografts with SP5-52Lipo-Dox. $A$, human lung cancer-bearing mice were injected i.v. with SP5-52-LipoDox, Lipo-Dox, or PBS. The tumor sizes of mice in the Lipo-Dox group and in the control PBS group were 1.9- and 4.1-fold larger than that of the SP5-52-Lipo-Dox group, respectively $\left(n=6 ;{ }^{*}, P<0.01\right)$ $B$, mice bearing lung cancer were treated with SP5-52-Lipo-Dox and Lipo-Dox (Kaplan-Meier survival curve). $C$, human oral cancer-bearing mice were injected i.v. with SP5-52-Lipo-Dox, Lipo-Dox, or PBS. SP5-52-Lipo-Dox significantly suppressed tumor growth $(n=6$; $\star, P<0.05)$. $D$, mice bearing oral cancers were treated with SP5-52-Lipo-Dox and Lipo-Dox (Kaplan-Meier survival curve). SP5-52-Lipo-Dox, SP5-52 peptideconjugated liposome-containing doxorubicin; Lipo-Dox, liposomes containing doxorubicin. 
therapy described here may overcome these problems by increasing the accumulation of drugs in the high IFP of the tumor through the affinity of the targeting ligand for tumor tissues.

In summary, using in vivo phage display to isolate ligands homing to molecules on tumor endothelial cells, we identified several peptides, including SP5-52, which targeted the neovasculature in two NSCLCs and six human tumor xenografts. Targeting liposomes via this peptide can inhibit the angiogenesis of tumors, resulting in increased therapeutic efficacy and higher survival rates of human lung and oral cancer xenograft mice. Therefore, SP5-52 is an effective agent for drug delivery to the neovasculature of solid tumors and may be suitable for application in clinical cancer treatment.

\section{Acknowledgments}

Received 6/15/2007; revised 9/13/2007; accepted 9/19/2007.

Grant support: National Science Council (NSC-95-2323-B-001-001) and Academia Sinica, Taiwan (H-C. Wu)

The costs of publication of this article were defrayed in part by the payment of page charges. This article must therefore be hereby marked advertisement in accordance with 18 U.S.C. Section 1734 solely to indicate this fact.

The authors thank Dr. Yun-Long Tseng for preparation of liposome complex and Mary Wyatt for reading the manuscript.

\section{References}

1. Bergers G, Song S, Meyer-Morse N, Bergsland E, Hanahan D. Benefits of targeting both pericytes and endothelial cells in the tumor vasculature with kinase inhibitors. J Clin Invest 2003;111:1287-95.

2. Ruoslahti E. Specialization of tumour vasculature. Nat Rev Cancer 2002;2:83-90.

3. Eliceiri BP, Cheresh DA. The role of $\alpha \mathrm{v}$ integrins during angiogenesis: insights into potential mechanisms of action and clinical development. J Clin Invest 1999;103: 1227-30.

4. Pasqualini R, Koivunen E, Ruoslahti E. $\alpha \mathrm{v}$ Integrins as receptors for tumor targeting by circulating ligands. Nat Biotechnol 1997;15:542-6.

5. Arap W, Pasqualini R, Ruoslahti E. Cancer treatment by targeted drug delivery to tumor vasculature in a mouse model. Science 1998;279:377-80.

6. Speth PA, van Hoesel QG, Haanen C. Clinical pharmacokinetics of doxorubicin. Clin Pharmacokinet 1988;15:15-31.

7. Drummond DC, Meyer O, Hong K, Kirpotin DB, Papahadjopoulos D. Optimizing liposomes for delivery of chemotherapeutic agents to solid tumors. Pharmacol Rev 1999;51:691-743.

8. Papahadjopoulos D, Allen TM, Gabizon A, et al. Sterically stabilized liposomes: improvements in pharmacokinetics and antitumor therapeutic efficacy. Proc Natl Acad Sci U S A 1991;88:11460-4.

9. Vaage J, Barbera-Guillem E, Abra R, Huang A, Working P. Tissue distribution and therapeutic effect of intravenous free or encapsulated liposomal doxorubicin on human prostate carcinoma xenografts. Cancer 1994;73: 1478-84.

10. Colbern GT, Hiller A, Musterer RS, Pegg E, Henderson IC, Working P. Significant increase in antitumor potency of doxorubicin $\mathrm{HCl}$ by its encapsulation in pegylated liposomes. J Liposome Res 1999;9:523-38.

11. Gabizon A, Catane R, Uziely B, et al. Prolonged circulation time and enhanced accumulation in malignant exudates of doxorubicin encapsulated in polyethylene-glycol coated liposomes. Cancer Res 1994; 54:987-92.

12. Marina NM, Cochrane D, Harney E, et al. Dose escalation and pharmacokinetics of pegylated liposomal doxorubicin (Doxil) in children with solid tumors: a Pediatric Oncology Group Study. Clin Cancer Res 2002;8: 413-8.

13. Safra T, Muggia F, Jeffers S, et al. Pegylated liposomal doxorubicin (doxil): reduced clinical cardiotoxicity in patients reaching or exceeding cumulative doses of $500 \mathrm{mg} / \mathrm{m}^{2}$. Ann Oncol 2000;11:1029-33.

14. Gabizon A, Martin F. Polyethylene glycol-coated (pegylated) liposomal doxorubicin. Rationale for use in solid tumours. Drugs 1997;54:15-21.

15. Dvorak HF, Nagy JA, Dvorak AM. Structure of solid tumors and their vasculature: implications for therapy with monoclonal antibodies. Cancer Cells 1991;3:77-85.

16. Shockley TR, Lin K, Nagy JA, Tompkins RG, Dvorak HF, Yarmush ML. Penetration of tumor tissue by antibodies and other immunoproteins. Ann N Y Acad Sci 1991;618:367-82.

17. Fu Y, Shearing LN, Haynes S, et al. Isolation from phage display libraries of single chain variable fragment antibodies that recognize conformational epitopes in the malaria vaccine candidate, apical membrane antigen-1. J Biol Chem 1997;272:25678-84.

18. Scott JK, Smith GP. Searching for peptide ligands with an epitope library. Science 1990;249:386-90.

19. Wu HC, Huang YL, Chao TT, et al. Identification of B-cell epitope of dengue virus type 1 and its application in diagnosis of patients. J Clin Microbiol 2001;39:977-82.

20. Wu HC, Jung MY, Chiu CY, et al. Identification of a dengue virus type 2 (DEN-2) serotype-specific B-cell epitope and detection of DEN-2-immunized animal serum samples using an epitope-based peptide antigen. J Gen Virol 2003;84:2771-9.

21. Atwell S, Ultsch M, De Vos AM, Wells JA. Structural plasticity in a remodeled protein-protein interface. Science 1997;278:1125-8.

22. Bottger V, Bottger A, Howard SF, et al. Identification of novel mdm2 binding peptides by phage display. Oncogene 1996;13:2141-7.

23. Nord K, Gunneriusson E, Ringdahl J, Stahl S, Uhlen $\mathrm{M}$, Nygren PA. Binding proteins selected from combinatorial libraries of an $\alpha$-helical bacterial receptor domain. Nat Biotechnol 1997;15:772-7.

24. Li B, Tom JY, Oare D, et al. Minimization of a polypeptide hormone. Science 1995;270:1657-60.

25. Wrighton NC, Farrell FX, Chang R, et al. Small peptides as potent mimetics of the protein hormone erythropoietin. Science 1996;273:458-64.

26. Castano AR, Tangri S, Miller JE, et al. Peptide binding and presentation by mouse CD1. Science 1995; 269:223-6.

27. Kraft S, Diefenbach B, Mehta R, Jonczyk A, Luckenbach GA, Goodman SL. Definition of an unexpected ligand recognition motif for $\alpha v \beta 6$ integrin. J Biol Chem 1999;274 1979-85.

28. Pasqualini R, Koivunen E, Ruoslahti E. A peptide isolated from phage display libraries is a structural and functional mimic of an RGD-binding site on integrins. J Cell Biol 1995;130:1189-96.

29. Folgori A, Tafi R, Meola A, et al. A general strategy to identify mimotopes of pathological antigens using only random peptide libraries and human sera. EMBO J 1994; 13:2236-43.

30. Prezzi C, Nuzzo M, Meola A, et al. Selection of antigenic and immunogenic mimics of hepatitis $\mathrm{C}$ virus using sera from patients. J Immunol 1996;156: 4504-13.

31. Liu IJ, Hsueh PR, Lin CT, et al. Disease-specific B cell epitopes for serum antibodies from patients with severe acute respiratory syndrome (SARS) and serologic detection of SARS antibodies by epitope-based peptide antigens. J Infect Dis 2004;190:797-809.

32. Barry MA, Dower WJ, Johnston SA. Toward celltargeting gene therapy vectors: selection of cell-binding peptides from random peptide-presenting phage libraries. Nat Med 1996;2:299-305.
33. Lee TY, Wu HC, Tseng YL, Lin CT. A novel peptide specifically binding to nasopharyngeal carcinoma for targeted drug delivery. Cancer Res 2004;64:8002-8.

34. Pasqualini R, Ruoslahti E. Organ targeting in vivo using phage display peptide libraries. Nature 1996;380 364-6.

35. Chu YW, Yang PC, Yang SC, et al. Selection of invasive and metastatic subpopulations from a human lung adenocarcinoma cell line. Am J Respir Cell Mol Biol 1997; 17:353-60.

36. Tseng YL, Hong RL, Tao MH, Chang FH. Sterically stabilized anti-idiotype immunoliposomes improve the therapeutic efficacy of doxorubicin in a murine B-cell lymphoma model. Int J Cancer 1999;80:723-30.

37. Habeeb AFSA. Determination of free amino groups in protein by trinitrobenzene sulfuric acid. Anal Biochem 1966;14:328.

38. Zalipsky S, Mullah N, Harding JA, Gittelman J, Guo L, DeFrees SA. Poly(ethylene glycol)-grafted liposomes with oligopeptide or oligosaccharide ligands appended to the termini of the polymer chains. Bioconjug Chem 1997;8:111-8.

39. Kirpotin D, Park JW, Hong K, et al. Sterically stabilized anti-HER2 immunoliposomes: design and targeting to human breast cancer cells in vitro. Biochemistry 1997;36:66-75.

40. Park JW, Hong K, Kirpotin DB, et al, Anti-HER2 immunoliposomes: enhanced efficacy attributable to targeted delivery. Clin Cancer Res 2002;8:1172-81.

41. Oku N, Asai T, Watanabe $\mathrm{K}$, et al. Anti-neovascular therapy using novel peptides homing to angiogenic vessels. Oncogene 2002;21:2662-9.

42. Hoffman JA, Giraudo E, Singh M, et al. Progressive vascular changes in a transgenic mouse model of squamous cell carcinoma. Cancer Cell 2003;4:383-91. 43. Boehm T, Folkman J, Browder T, O'Reilly MS. Antiangiogenic therapy of experimental cancer does not induce acquired drug resistance. Nature 1997;390: 404-7.

44. Denekamp J. Angiogenesis, neovascular proliferation and vascular pathophysiology as targets for cancer therapy. Br J Radiol 1993;66:181-96.

45. Burrows FJ, Thorpe PE. Vascular targeting: a new approach to the therapy of solid tumors. Pharmacol Ther 1994;64:155-74.

46. Heldin $\mathrm{CH}$, Rubin $\mathrm{K}$, Pietras K, Ostman A. High interstitial fluid pressure-an obstacle in cancer therapy. Nat Rev Cancer 2004;4:806-13.

47. Less JR, Posner MC, Boucher Y, Borochovitz D, Wolmark N, Jain RK. Interstitial hypertension in human breast and colorectal tumors. Cancer Res 1992;52:6371-4.

48. Boucher Y, Kirkwood JM, Opacic D, Desantis M, Jain $\mathrm{RK}$. Interstitial hypertension in superficial metastatic melanomas in humans. Cancer Res 1991;51:6691-4. 49. Gutmann R, Leunig $M$, Feyh $J$, et al. Interstitial hypertension in head and neck tumors in patients: correlation with tumor size. Cancer Res 1992;52:1993-5. 50. Boucher Y, Baxter LT, Jain RK. Interstitial pressure gradients in tissue-isolated and subcutaneous tumors: implications for therapy. Cancer Res 1990;50:4478-84. 